\title{
Non-invasive prenatal diagnosis of spinal muscular atrophy by relative haplotype dosage
}

\author{
Michael Parks ${ }^{\star,}$, Samantha Court ${ }^{1}$, Benjamin Bowns ${ }^{1}$, Siobhan Cleary ${ }^{1}$, Samuel Clokie ${ }^{1}$, Julie Hewitt ${ }^{2}$, \\ Denise Williams ${ }^{2}$, Trevor Cole ${ }^{2}$, Fiona MacDonald ${ }^{1}$, Mike Griffiths ${ }^{1}$ and Stephanie Allen ${ }^{1}$
}

\begin{abstract}
Although technically possible, few clinical laboratories across the world have implemented non-invasive prenatal diagnosis (NIPD) for selected single-gene disorders, mostly owing to the elevated costs incurred. Having previously proven that NIPD for X-linked disorders can be feasibly implemented in clinical practice, we have now developed a test for the NIPD of an autosomalrecessive disorder, spinal muscular atrophy (SMA). Cell-free DNA was extracted from maternal blood and prepared for massively parallel sequencing on an Illumina MiSeq by targeted capture enrichment of single-nucleotide polymorphisms across a $6 \mathrm{Mb}$ genomic window on chromosome 5 containing the SMN1 gene. Maternal, paternal and proband DNA samples were also tested for haplotyping purposes. Sequencing data was analysed by relative haplotype dosage (RHDO). Six pregnant SMA carriers and 10 healthy pregnant donors were recruited through the NIPSIGEN study. Inheritance of the maternally and paternally derived alleles of the affected SMN1 gene was determined in the foetus by RHDO analysis for autosomal-recessive disorders. DNA from the proband (for SMA carriers) or an invasively obtained foetal sample (for healthy pregnant donors) was used to identify the maternal and paternal reference haplotypes associated with the affected SMN1 gene. Results for all patients correlated with known outcomes and showed a testing specificity and sensitivity of $100 \%$. On top of showing high accuracy and reliability throughout the stages of validation, our novel test for NIPD of SMA is also affordable and viable for implementation into clinical service.
\end{abstract}

European Journal of Human Genetics (2017) 25, 416-422; doi:10.1038/ejhg.2016.195; published online 25 January 2017

\section{INTRODUCTION}

The presence of cell-free foetal DNA (cffDNA) in maternal plasma during pregnancy was first described in 1997. ${ }^{1}$ Apoptosis of placental trophoblasts releases small fragments of cffDNA, which enter the maternal circulation. ${ }^{2}$ These fragments comprise approximately $10 \%$ of the total cell-free DNA (cfDNA) in maternal blood, ${ }^{3,4}$ with the remainder being maternally derived. The discovery of cffDNA presented significant opportunities in terms of prenatal diagnostics. Methodologies based around the detection of paternally inherited sequences are well established, with clinical uses including foetal sexing ${ }^{5-7}$ and $\mathrm{RhD}$ blood group genotyping. ${ }^{8-10}$ Advances in massively parallel sequencing (MPS) technologies have led to the development of non-invasive prenatal testing (NIPT) for aneuploidy screening, ${ }^{11-15}$ which is now an established service in many countries worldwide. ${ }^{16}$ Methods for the non-invasive prenatal diagnosis (NIPD) of singlegene disorders (SGDs) have also been developed ${ }^{17,18}$ but have been mostly limited to the detection of paternally inherited ${ }^{19-21}$ and de novo mutations. ${ }^{22}$ However, recent studies have proven that MPS-based approaches for NIPD of SGDs, including $\beta$-thalassemia, ${ }^{23}$ congenital adrenal hyperplasia $(\mathrm{CAH})^{24,25}$ and Duchenne and Becker muscular dystrophies (DMD/BMD), ${ }^{26}$ are technically possible but prohibitively expensive when considering clinical implementation.

Our group recently published the results of a study conducted at the Birmingham Women's NHS Foundation Trust (UK) as part of the NIPSIGEN project (Non-Invasive Prenatal diagnosis for Single Gene disorders) aimed at the development and clinical implementation of affordable NIPD tests for SGDs. Our method, which uses capturebased targeted enrichment followed by MPS and analysis by relative haplotype dosage (RHDO), ${ }^{27}$ enabled the highly accurate NIPD of $\mathrm{DMD} / \mathrm{BMD}$ in at-risk pregnancies at an affordable cost for clinical implementation. ${ }^{28}$ We have now extended this method to encompass NIPD of spinal muscular atrophy (SMA), with similarly promising results.

SMA is an autosomal-recessive neurodegenerative disease with variable expression, caused by deletions, gene conversions or mutations in the SMN1 gene. ${ }^{29,30}$ With an incidence of around 1 in 10000 live births, it is the second most common autosomal-recessive disorder after cystic fibrosis in the northern European population. ${ }^{31}$ SMN1 maps to a complex region of chromosome $5 \mathrm{q} 13.1$, where an element of around $500 \mathrm{~kb}$ containing four genes (SMN, NAIP, SERF and GTFH2) is present in inverted duplicate copies. SMN1, located in the telomeric copy, is almost identical to the pseudogene SMN2, located in the centromeric copy, except for five nucleotide changes in exons 7 and $8 .^{30}$ Causative mutations are divided into three categories: (1) homozygous deletion of SMN1 or gene conversion from SMN1 to SMN2 (approximately 95\% of SMA cases); (2) compound heterozygosity involving deletion/gene conversion of one copy of SMN1 with a nonsense, frameshift or missense mutation in the remaining copy (approximately 5\% of cases); and (3) homozygous or compound heterozygous mutation of SMN1 (very rare). ${ }^{31}$ Prenatal

${ }^{1}$ West Midlands Regional Genetics Laboratory, Birmingham Women's NHS Foundation Trust, Birmingham, UK; ${ }^{2}$ West Midlands Regional Genetics Service, Birmingham Women's NHS Foundation Trust, Birmingham, UK

*Correspondence: Dr M Parks, West Midlands Regional Genetics Laboratory, Birmingham Women's NHS Foundation Trust, Mindelsohn Way, 97, Vincent Drive, Edgbaston, Birmingham, B15 2TG, UK. Tel: +44 0121607 4776; Fax: +44 0121627 2711; E-mail: michael.parks@bwnft.nhs.uk Received 9 June 2016; revised 2 November 2016; accepted 22 November 2016; published online 25 January 2017 
diagnosis of at-risk pregnancies currently involves the analysis of foetal DNA obtained by invasive procedures, such as chorionic villus sampling (CVS) or amniocentesis. SMN1 copy number can be determined by methods, including restriction fragment length polymorphism testing, multiplex ligation-dependent probe amplification (MLPA) or quantitative PCR. ${ }^{31}$ However, this type of analysis is limited in cases where two or more copies of SMN1 are present in cis on the same chromosome in combination with SMN1 deletion/gene conversion on the other chromosome, which accounts for around $4 \%$ of SMA carriers. ${ }^{32}$ Other intragenic mutations can be detected by sequencing or linkage analysis using informative markers flanking SMN1. ${ }^{31}$ Obtaining samples by these types of invasive techniques is the only diagnostic option currently available and is associated with a $0.5-1 \%$ risk of miscarriage. ${ }^{33,34}$ An alternative non-invasive strategy would eliminate this risk and allow testing to be carried out at an earlier point in gestation. Our method for NIPD of SMA has shown promising results during validation testing, with a level of affordability conducive to clinical implementation.

\section{SUBJECTS AND METHODS}

\section{Patients and samples}

Patients were recruited into two separate groups through the NIPSIGEN study ('NIPSIGEN: clinical translation of NIPD for SGDs'; REC approval number: 13/ NW/0580). Group 1 included pregnant women referred owing to risk of foetal aneuploidy who were offered invasive prenatal testing at West Midlands Regional Genetics Laboratory. Blood samples from women and their partners in this group were used as validation controls to assess the efficiency, accuracy and multiplexing capacity of our method. NIPD testing by RHDO can be performed on these patients by using the foetal genomic DNA obtained through invasive sampling to determine the reference haplotypes. Pregnant couples who are known carriers of SMA mutations were recruited nationwide (UK) to group 2. For these patients, we requested a sample of genomic DNA from a previous affected child to use as proband in determining the haplotypes linked with the paternal and maternal mutant alleles. The DNA samples needed for each patient included the cfDNA extracted from maternal plasma, the maternal and paternal genomic DNA extracted from leukocytes and the proband genomic DNA (from the invasively obtained sample for group 1 patients and from a previous affected child for group 2 patients). A maximum of 12 samples (ie, up to three patients) were processed simultaneously and pooled together prior to targeted capture enrichment and MPS. cfDNA was extracted from $4 \mathrm{ml}$ of plasma and eluted in a final volume of $60 \mu \mathrm{l}$. Maternal genomic DNA was extracted from the leukocytes contained in $1 \mathrm{ml}$ of the blood cell portion, with paternal genomic DNA extracted in the same manner. Foetal genomic DNA was extracted from enzymatically digested CVS/amniotic fluid material and eluted into a final volume of $50 \mu$ l. Proband DNA samples were extracted externally. Full details on sample processing and DNA extraction can be found in Supplementary Appendix SA.

\section{Targeted MPS}

DNA libraries for MPS on the MiSeq sequencing platform (Illumina, Inc., San Diego, CA, USA) were prepared from 29 to $122 \mathrm{ng}$ of input DNA. Capture enrichment was designed to target highly heterozygous single-nucleotide polymorphisms (SNPs) across the SMN1/SMN2 gene region (Chr 5: 67000 53072999 964). Up to 12 samples (equivalent to up to three patients) were multiplexed per sequencing run using $2 \times 80$ cycles paired-end settings. More details can be found in Supplementary Appendix SB. Bioinformatics analysis included quality trimming of reads, alignment to genome build hg19, removal of duplicates and variant calling to obtain SNP counts (Supplementary Appendix SC).

\section{Data analysis by RHDO}

RHDO analysis for autosomal-recessive disorders has been successfully used in proof-of-concept studies for NIPD of $\beta$-thalassemia ${ }^{23}$ and $\mathrm{CAH} .{ }^{24}$ In this study, we have applied RHDO analysis for NIPD of SMA in a similar manner. Briefly, both maternal and paternal haplotypes linked with the mutant alleles were identified by DNA sequencing of highly heterozygous bi-allelic SNPs on both sides of the SMN1 gene (as previously described) in a previous affected child. The genotypes of the same SNPs were determined in maternal and paternal DNA samples to conduct haplotype phasing and identify the haplotypes linked with the normal alleles. SNP counts obtained from cfDNA sequencing were used to determine whether the foetus had inherited the mutated (M-ma) or normal $(\mathrm{N}-\mathrm{ma})$ maternal alleles and the mutated (M-pa) or normal (N-pa) paternal alleles. The paternally inherited haplotype was identified by using SNPs that were homozygous in the mother and heterozygous in the father. The maternally inherited haplotype was determined by RHDO analysis conducted on SNPs that were heterozygous in the mother and homozygous in the father. In this instance, SNPs had to be further subdivided into $\alpha$ (where the paternal SNP allele matched the maternal SNP allele on the affected haplotype) and $\beta$ SNPs (where the paternal SNP allele matched the maternal SNP allele on the normal haplotype) and were analysed separately. The foetal fraction was determined by using SNPs that were homozygous in both parents but for different SNP alleles (Supplementary Appendix SD). Informative SNPs used to determine maternal and paternal inheritance were separately grouped into haplotype blocks of $\geq 25$ SNPs to form maternal $\alpha$ and $\beta$ blocks and paternal blocks. Each haplotype block represents a statistically independent result. Data quality filters and RHDO analysis parameters were adopted from previous publications $\mathrm{s}^{24,28}$ (Supplementary Appendix SDinformation).

\section{MLPA analysis}

Routine invasive prenatal diagnosis of SMA patients was conducted using MLPA analysis to detect copy number of exons 7 and 8 in the SMN1 and SMN2 genes (SALSA MLPA P021 SMA kit, sold by MRC-Holland (Amsterdam, The Netherlands)). The genomic coordinates of the exons tested are the following: SMN1 exon 7: chr5.hg19:g.70 247 768-70 247 821; SMN1 exon 8: chr5.hg19:g.70 248 266-70 248 839; SMN2 exon 7: chr5.hg19:g.69 $372348-$ 69372 401; SMN2 exon 8: chr5.hg19:g.69 372 846-69 373422.

\section{RESULTS}

\section{Probe selection criteria for efficient SNP enrichment}

Targeted enrichment by probe capture prior to MPS has been successfully used for copy number variation tests in combination with RHDO analysis in previous studies. ${ }^{23,24}$ More recently, we were successful in further developing this approach by designing a highly efficient probe library for the targeted capture of $>1000$ SNPs across the dystrophin gene for NIPD testing of DMD/BMD. ${ }^{28}$ Using the same criteria, we selected 3039 SNPs from dbSNP(144) with a reported average heterozygosity of $\geq 40 \%$ across a $6 \mathrm{Mb}$ region on chromosome 5 (Chr5: 67000 530-72 999 964) containing the SMN1 and SMN2 genes (1000 genomes data). Probes were then specifically designed to uniquely target the selected SNPs. A highly repetitive region exists within the targeted $6 \mathrm{Mb}$ (see Figure 1); therefore, SNPs within this range (Chr5: 68813 676-70 680 481) were excluded from the probe design. The overall captured area was $276 \mathrm{~Kb}$ and SNPs were evenly distributed between the centromeric (1486 SNPs) and telomeric (1553 SNPs) regions flanking the SMN1 and SMN2 genes.

\section{Initial validation on group 1 patients: pregnancies A-J}

Samples of healthy patients undergoing prenatal diagnosis for aneuploidy testing owing to increased risk (recruited locally to group 1) were initially used as controls to ascertain the efficiency, accuracy and multiplexing capacity of the test. Blood samples from the 10 patients tested were taken between 11 and 15 weeks of gestation. Details on outcomes and testing parameters are summarised in Table 1. On average, we observed a foetal fraction of $10.93 \%$ (ranging between 6.83 and 20.31\%) and identified 632 informative SNPs per patient (interquartile range $(\mathrm{IQR})=250$ ) after quality filtering. The quantity of haplotype blocks identified for maternal inheritance ranged from 3 


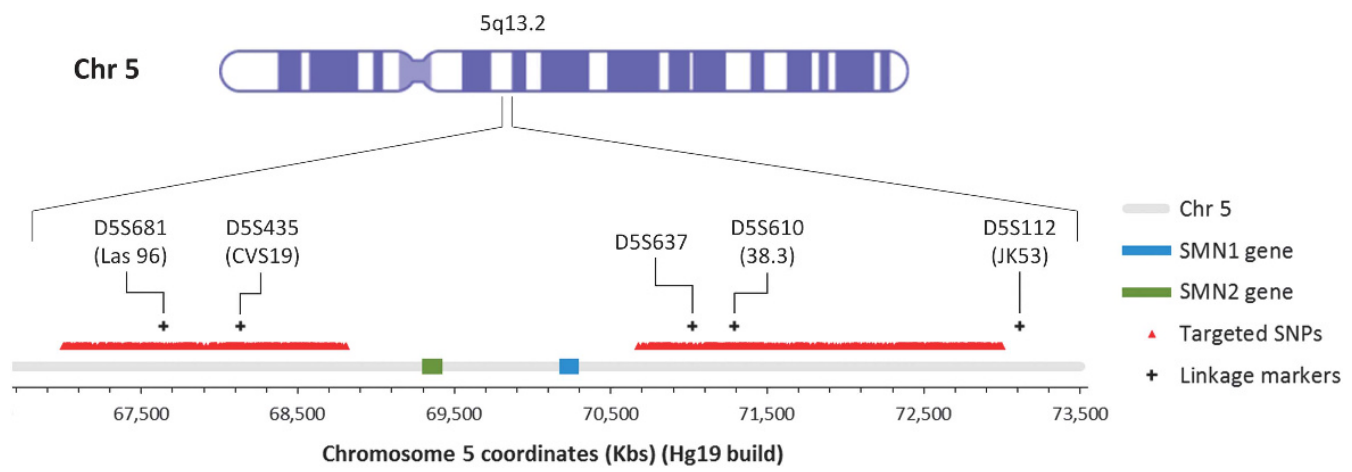

Figure 1 Diagram of the $5 q 13.2$ locus on chromosome 5 containing the SMN1 and SMN2 genes represented by the light blue and green boxes, respectively. The red triangles indicate the chromosome position of SNPs with AvHet $>0.4$, which were targeted through capture-based DNA library enrichment for NIPD of SMA. The black crosses indicate the position of the markers routinely used in our laboratory for linkage analysis in SMA families.

Table 1 Summary of tests conducted on patients from group 1 (pregnancies A-J)

\begin{tabular}{|c|c|c|c|c|c|c|c|c|}
\hline Pregnancies & $\begin{array}{l}\text { Outcome } \\
\text { (mat/pat) }\end{array}$ & Gestation & $\begin{array}{l}\text { Foetal } \\
\text { fraction }\end{array}$ & $\begin{array}{l}\text { Reference } \\
\text { haplotypes }\end{array}$ & $\begin{array}{l}\text { Informative } \\
\text { SNPs used }\end{array}$ & $\begin{array}{l}\text { Haplotype blocks } \\
\text { (mat/pat) }\end{array}$ & $\begin{array}{c}\text { Classification } \\
\text { accuracy }\end{array}$ & $\begin{array}{c}\text { Average sequencing depth of infor- } \\
\text { mative SNPs used }\end{array}$ \\
\hline$A$ & НарA-HapA & 13 weeks+4 days & $14.34 \%$ & CVS & 823 & $18 / 8$ & $100 \%$ & 339 \\
\hline$B$ & НарА-НарА & 13 weeks +5 days & $7.56 \%$ & CVS & 898 & $20 / 12$ & $100 \%$ & 321 \\
\hline C & НарA-HapA & 14 weeks +5 days & $9.83 \%$ & CVS & 726 & $18 / 10$ & $100 \%$ & 259 \\
\hline D & НарA-HapA & 13 weeks+1 day & $7.51 \%$ & CVS & 811 & $13 / 11$ & $100 \%$ & 270 \\
\hline$E$ & HapA-HapA & 11 weeks +2 days & $20.31 \%$ & CVS & 566 & $14 / 8$ & $100 \%$ & 223 \\
\hline $\mathrm{F}$ & НарА-НарА & 15 weeks+5 days & $6.83 \%$ & AMNIO & 675 & $10 / 10$ & $95 \%$ & 257 \\
\hline G & НарА-НарА & 13 weeks+5 days & $11.69 \%$ & CVS & 653 & $14 / 10$ & $100 \%$ & 189 \\
\hline $\mathrm{H}$ & НарА-НарА & 11 weeks+1 day & $12.21 \%$ & CVS & 531 & $11 / 9$ & $100 \%$ & 149 \\
\hline I & НарА-НарА & 11 weeks+3 days & $6.91 \%$ & CVS & 278 & $3 / 5$ & $100 \%$ & 89 \\
\hline J & НарА-НарА & 14 weeks & $12.10 \%$ & CVS & 360 & $9 / 4$ & $100 \%$ & 64 \\
\hline
\end{tabular}

Outcomes are reported as paternal and maternal inheritance of the reference haplotypes (HapA) identified in the foetal DNA obtained by invasive sampling or the opposite haplotypes (HapB) identified in the maternal and paternal DNA samples by haplotype phasing. Only informative SNPs that passed the quality filtering criteria (see Supplementary Appendix A) were used for data analysis. The numbers of haplotype blocks identified for paternal and maternal inheritance are kept separate. The classification accuracy represents the percentage of haplotype blocks, which showed an expected inheritance pattern. The average sequencing depth has been calculated on the informative SNPs used for data analysis.

to 20, while haplotype blocks identified for paternal inheritance ranged from 4 to 12 . Overall, $216 / 217$ haplotype blocks were correctly classified, resulting in a testing accuracy of $99.53 \%$. For these patients, the maternal and paternal reference haplotypes identified from the corresponding DNA sample obtained by CVS or amniocentesis were classified as HapA-ma and HapA-pa, respectively. The alternative haplotypes identified by phasing in maternal and paternal DNA samples were classified as HapB-ma and HapB-pa. Therefore, we expected to observe an outcome of HapA-ma/HapA-pa in all tested pregnancies of group 1 patients, which was indeed the case. The results reported in Table 1 demonstrate that the test was capable of delivering high accuracy and consistency for group 1 patients, with an overall sensitivity and specificity of $100 \%$ and a maximum multiplexing capacity of three patients per sequencing run (MiSeq sequencing platform (Illumina, Inc.). A single maternal haplotype block was incorrectly classified in family $\mathrm{F}$ but did not affect the final result. Incorrect classification of haplotype blocks in RHDO analysis has been previously reported at an estimated rate of $<2 \%{ }^{27}$ and can be caused by low foetal fraction and underlying biological factors. Therefore, a switch in inheritance pattern observed in a single haplotype block is disregarded and does not affect the final result. However, two or more consecutive haplotype blocks showing a switch in inheritance pattern indicate the presence of a recombination event, ${ }^{24,27,28}$ which needs to be taken into account in the final result.

\section{Results for group 2 patients: families $\mathrm{K}-\mathrm{O}$}

Testing of six pregnancies in five patients at risk of carrying a child affected with SMA was conducted for clinical validation. In all cases, the haplotypes linked to the SMA mutations were identified from the DNA sample of a previous affected sibling of the foetus. A summary of results and testing parameters is presented in Table 2, and a graphical representation of maternally and paternally inherited haplotype blocks is illustrated in Figure 2 for each pregnancy. In four of the five patients tested (families K, L, M and N), both parents were confirmed carriers with one copy of exons 7 and 8 in the SMN1 gene detected by MLPA analysis. In family $\mathrm{O}$, only the mother was confirmed as a carrier of SMA (see following section). NIPD of SMA showed an unaffected outcome in family $\mathrm{K}$, carrier status in family L (paternally inherited), carrier status in family $\mathrm{M}$ (paternally inherited), affected outcome in family $\mathrm{N}$ and an affected and carrier (maternally inherited) results, respectively, for the first $(\mathrm{P} 1)$ and second $(\mathrm{P} 2)$ pregnancies in family O. All outcomes were confirmed by MLPA analysis on CVS samples, except for the second pregnancy in family O (P2) (see following section). Testing performances for these patients matched closely with the data obtained from group 1 patients, with an average number of 
644 informative SNPs identified $(\mathrm{IQR}=124)$ after quality filtering. Foetal fraction ranged from 9.09 to $16.49 \%$ with an average of $11.75 \%$; and the accuracy of haplotype block classification was $99.26 \%$ (135/136), with only one haplotype block incorrectly classified in family K. This data prove the clinical validity of our NIPD test for SMA, which reported a sensitivity and specificity rate of $100 \%$ for patients tested with no inconclusive results and a failure rate of $0 \%$.

\section{Advantages of NIPD over invasive testing in family $\mathbf{O}$}

The parents in family $\mathrm{O}$ were screened for SMA carrier status after their second child was diagnosed with the disease (Figure 3). The mother was found to be carrying one copy of exons 7 and 8 in the SMN1 gene, as detected by MLPA analysis. The carrier status of the father, however, could not be confirmed, as he was found to have two copies of exons 7 and 8 for SMN1. Therefore, it was hypothesized that the father may be carrying two copies of SMN1 on one allele and none on the other, as has been proven to happen in around $4 \%$ of cases. ${ }^{32}$ Alternatively, the father may have been a carrier for a mosaic germline mutation or there may have been a de novo mutation on one allele in the affected child. NIPD testing on the patient's subsequent pregnancy (P1) using the DNA sample from the previous affected sibling of the foetus to identify the haplotypes linked with the mutant alleles yielded an affected result (Figure 2). The outcome was confirmed by MLPA analysis conducted on the CVS sample and indicated, therefore, that the father was likely to be a carrier of SMA. In the following pregnancy (P2), NIPD testing revealed that the foetus was a carrier, having inherited the mutated haplotype from the mother (M-ma) and the normal haplotype from the father (N-pa; Figure 2). In this case, MLPA analysis conducted on the invasively obtained foetal sample yielded an unaffected result, having detected two copies of exons 7 and 8 in the SMN1 gene. Taken together, this data prove that the father is indeed a carrier of SMA and has two copies of SMN1 on one allele and none on the other, as previously hypothesized. In summary, NIPD testing of SMA in this family provided a more informative result by confirming the carrier status of the second tested pregnancy (P2). Although the foetus would have still been correctly diagnosed as unaffected by invasive testing, knowledge of its carrier status holds important implications for the baby's future reproductive possibilities. However, it is important to highlight that, in cases where carrier status is not confirmed for one of the parents, a confirmatory invasive test would need to be conducted for affected outcomes in which the high-risk haplotype has been identified by NIPD testing. This is because we are testing for a haplotype and not the mutation directly. Therefore, pregnancy P1 would have been reported as 'increased risk' rather than 'affected' in a clinical setting, and an invasive test would have been warranted to confirm the 'affected' outcome.

\section{DISCUSSION}

The implementation of NIPD tests for SGDs into clinical practice has been slow and limited. By comparison, foetal sexing and $\mathrm{RhD}$ typing using cffDNA are now in routine clinical service ${ }^{11}$ in many countries across Europe. Furthermore, non-invasive aneuploidy screening tests developed by several commercial companies ${ }^{13,35-37}$ are being introduced in public health services worldwide, ${ }^{16}$ yet only a few centres are offering NIPD for SGDs. In the United Kingdom, a number of NIPD tests for the detection of paternally inherited and de novo mutations in selected SGDs have been developed as part of the RAPID project, ${ }^{21,22}$ some of which are now available as a clinical service. ${ }^{11}$ Although various studies have demonstrated that comprehensive NIPD testing for certain SGDs is technically possible, ${ }^{23-27}$ challenges pertaining to the high testing costs incurred remain to be addressed before clinical 


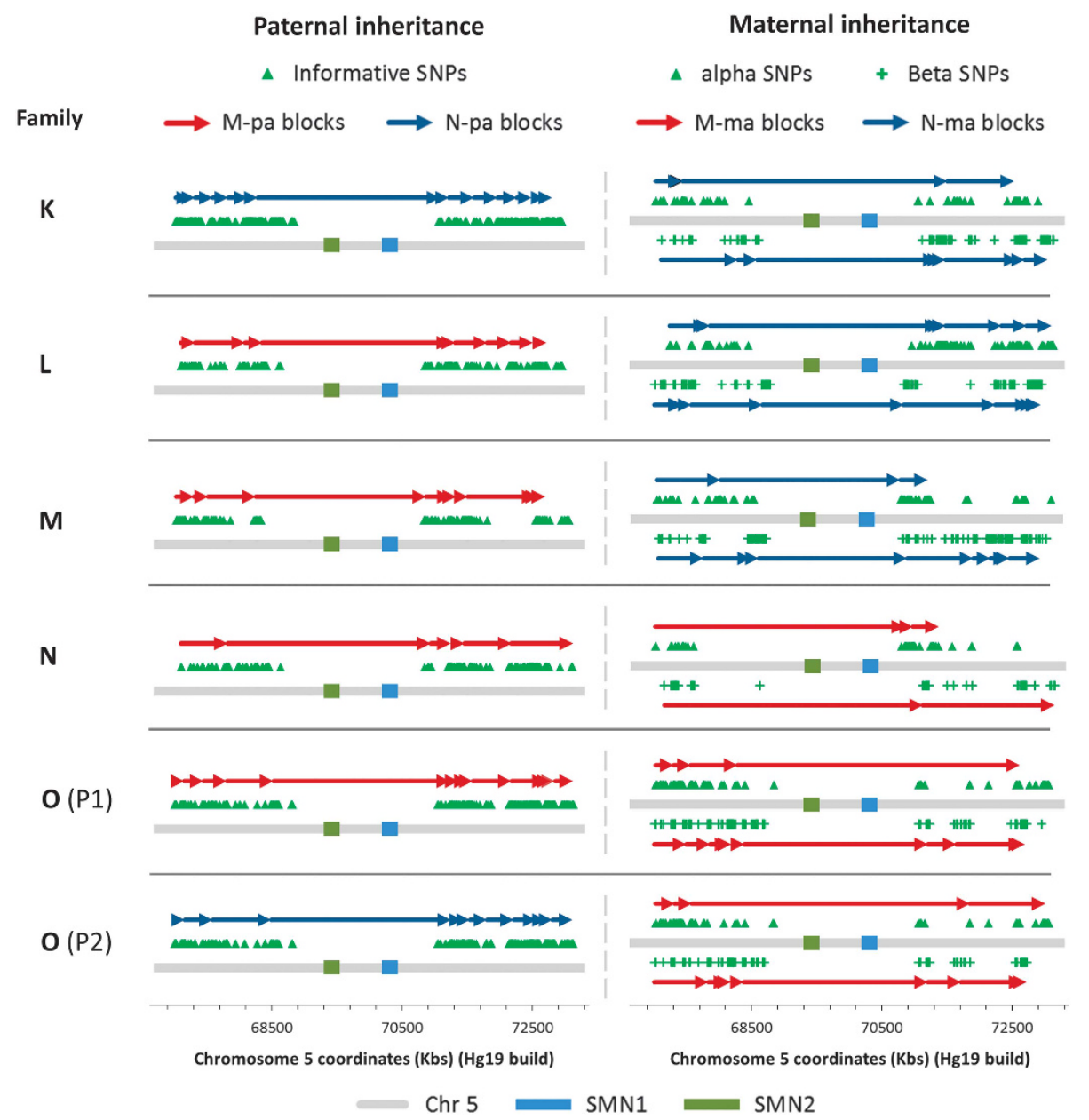

Figure 2 Graphic representation of NIPD results for patients at risk of SMA (families K-0) subdivided by foetal inheritance of paternal and maternal haplotypes. Haplotype blocks are represented by contiguous arrows spanning a $6 \mathrm{Mb}$ genomic window on chromosome 5 (grey line) containing the SMN1 (blue box) and SMN2 (green box) genes. Red arrows indicate that the foetus has inherited the mutated allele (M-pa from the father; and M-ma from the mother), while blue arrows indicate that the normal allele has been inherited ( $\mathrm{N}$-pa from the father; and $\mathrm{N}$-ma from the mother). The position of informative SNPs used to identify haplotype blocks is shown for both paternal (green triangles) and maternal (green triangles for $\alpha$ SNPs; green crosses for $\beta$ SNPs) inheritance.

implementation can be achieved. ${ }^{24,26}$ Recently, our team at Birmingham Women's NHS Foundation Trust has succeeded in developing an affordable and clinically feasible NIPD test for DMD/BMD through the NIPSIGEN project. ${ }^{28}$ Building on our experience, we have endeavoured to develop similar tests for other common autosomalrecessive disorders. In this study, we describe a new method for the NIPD of SMA by RHDO analysis capable of delivering accurate and informative results, as well as the foetus' SMA carrier status, irrespective of the SMA mutation profile present in the parents. This is an advantage in cases where copy number analysis of the SMN1 gene in carrier screening and prenatal tests is unable to confirm carrier status owing to a parental allele carrying two copies of the gene. However, in these cases NIPD tests revealing an affected outcome would need to be confirmed by an invasive test to avoid the possibility of a false positive result owing to germline mosaicism in the parent with unconfirmed carrier status or the possibility of a de novo mutation.

Overall, the data obtained from all the 13 patients tested, of which six pregnancies at risk of SMA, has shown sensitivity and specificity rates of $100 \%$, with $0 \%$ failure rate. The accuracy of our method in correctly classifying haplotype blocks amounted to $99.43 \%$ (351/353).
This reflects the reliability of the test, as each haplotype block represents a statistically independent result. Although we did not detect any recombination events across the targeted region containing the SMN1 and SMN2 genes in patients tested so far, the numbers of haplotype blocks identified for both paternal and maternal inheritance led us to believe that our method would be capable of detecting recombination sites with high accuracy. However, this would not apply to recombination events occurring within the highly repetitive genomic region on chromosome 5 located around the SMN1 and SMN2 genes (chromosome coordinates: 68813676 and 70680481 ) (Figure 1), where we were unable to design unique probes for targeted enrichment of selected SNPs. Although the possibility of this event is $<1 \%,{ }^{38}$ an inconclusive result would be reported in these cases and prenatal diagnosis by invasive testing would be warranted. Additionally, our NIPD test is not applicable in dizygotic twin pregnancies and in the absence of a viable DNA sample from an affected sibling. There also remains a very small risk of misdiagnosis in the event of a vanishing twin or in the presence of a double recombination within the highly repetitive region containing the SMN1 and SMN2 genes where we were unable to design SNP probes. Other limitations of our NIPD test include the common limitations associated with cffDNA 


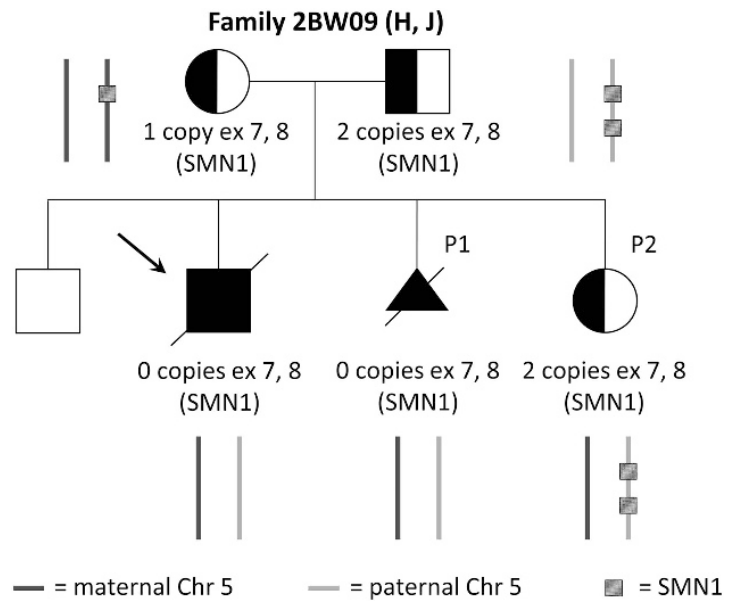

Figure 3 Family tree of family O. Copy number status of the SMN1 gene is reported for family members affected by/carriers of SMA, as reported by MLPA analysis. This is graphically illustrated by showing the copies of SMN1 gene for both maternal (black) and paternal (grey) alleles on the side. A DNA sample from the previous affected son (black arrow) was used to identify the maternal and paternal haplotypes linked with the mutant alleles. NIPD testing for SMA was carried out on the subsequent pregnancies (P1 and P2).

analysis, such as the impossibility of obtaining a viable diagnostic result in the presence of maternal somatic mosaicism or if the patient has undergone transplant surgery (ie, is the recipient of a donor organ). Low foetal fraction in cfDNA is also a limitation to NIPD testing, with $4 \%$ foetal fraction being generally considered as the cutoff limit. ${ }^{39-41}$ Nevertheless, data published in a previous study ${ }^{24}$ and generated in our laboratory (data not shown) led us to believe that a foetal fraction of $2 \%$ would reflect a more accurate cutoff limit for NIPD testing by RHDO analysis. In fact, the quantity of informative SNPs used for data analysis ensures that a statistically significant result is reached even in the presence of low foetal fractions. This, however, might not be the case for pregnancies in consanguineous couples, where the quantity of informative SNPs usable for RHDO analysis would be significantly reduced. In these cases, SNPs that are heterozygous in both parents would become informative and could be used to determine the maternally and paternally inherited haplotypes, ${ }^{23}$ although further testing on consanguineous couples would be required to validate this analysis method for clinical use.

Implementation of NIPD testing for SGDs into clinical practice has been mainly hindered by the prohibitive testing costs incurred, ${ }^{24,26}$ which cannot be curbed by increasing the patient multiplexing capacity of the test owing to the small number of couples who would request it. In this respect, the method presented in this study is capable of delivering accurate NIPD of SMA at an arguably reasonable cost. This is mainly achieved by designing highly efficient capture probes for targeted enrichment, which enables the use of lower-cost MiSeq sequencing platform (Illumina, Inc.) sequencing platform; by being able to multiplex up to three patients on the same sequencing run for up to three different SGDs; and by testing maternal, paternal and proband samples alongside the cfDNA sample to reduce haplotyping costs. Taking these considerations into account, we have calculated the

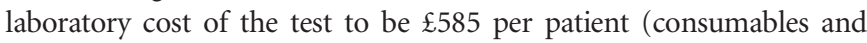
staff costs only) when multiplexing three patients. However, the full cost would need to take into account equipment costs and additional overheads. An additional cost that is impossible to quantify is the influence that NIPD testing might have on patient choice. Cost to a health service would potentially be increased by couples requesting non-invasive tests, which they would not have considered pursuing by invasive techniques. This needs to be weighed against the increased number of positive prenatal diagnoses and the financial impact of subsequent management decisions. Furthermore, consideration needs to be given to the quality improvement gained from increased patient choice and avoidance of procedure-related miscarriage. We therefore believe the final cost of the test to be acceptable for clinical use in the United Kingdom and are in the process of seeking approval from the UK Genetic Testing Network (UKGTN) to recommend national commissioning within the NHS. By running the test on a weekly basis with a turn-around time of 7-10 working days (depending on the day of the week the blood sample is received), we aim to provide the patient with a final report within the first trimester of pregnancy, as the blood sample can be taken as early as 8 weeks' gestation. Therefore, our NIPD method will not only deliver a safer test by eliminating the risk of miscarriage linked with invasive sampling procedures, thus improving quality of service, but will also provide patients with more time to manage their pregnancy.

In summary, having previously proved that NIPD of an X-linked condition can be achieved by RHDO analysis in a clinical setting, ${ }^{28}$ we here provide evidence that the same approach can be applied to autosomal-recessive disorders. Initial validation of our NIPD test for SMA has shown promising results, with an overall sensitivity and specificity of $100 \%$ on 16 patients tested to date. Given our success in using RHDO analysis for NIPD of SGDs, we intend to implement such testing into clinical practice within the United Kingdom and widen our testing repertoire, with the aim of enabling our patients to access swift and safe NIPD testing for a variety of Mendelian disorders.

\section{CONFLICT OF INTEREST}

The authors declare no conflict of interest.

\section{ACKNOWLEDGEMENTS}

This publication presents independent research commissioned by the Health Innovation Challenge Fund (HICF-R6-381), a parallel funding partnership between the Wellcome Trust and the Department of Health. The views expressed in this publication are those of the author(s) and not necessarily those of the Wellcome Trust or the Department of Health. Research was conducted at the West Midlands Regional Genetics Laboratory, part of Birmingham Women's NHS Foundation Trust. We thank all patients and donors for providing us with the blood samples for this study and our collaborators nationwide for help in recruitment. We also thank Kelly Hard in our R\&D team for helping us obtain ethical approval for the study and Dr Helen White, Ian Newington, Sally Nicholas and Rosalind Skinner in our steering committee for support and advice.

1 Lo YM, Corbetta N, Chamberlain PF et al: Presence of fetal DNA in maternal plasma and serum. Lancet 1997; 350: 485-487.

2 Alberry $M$, Maddocks $D$, Jones $M$ et al: Free fetal DNA in maternal plasma in anembryonic pregnancies: confirmation that the origin is the trophoblast. Prenat Diagn 2007; 27: 415-418.

3 Lun FMF, Chiu RWK, Chan KCA, Leung TY, Lau TK, Lo YMD: Microfluidics digital PCR reveals a higher than expected fraction of fetal DNA in maternal plasma. Clin Chem 2008; 54: 1664-1672.

4 Barrett AN, Zimmermann BG, Wang D, Holloway A, Chitty LS: Implementing prenatal diagnosis based on cell-free fetal DNA: accurate identification of factors affecting fetal DNA yield. PLoS One 2011; 6: e25202.

5 Rijnders RJ, van der Schoot CE, Bossers B, de Vroede MA, Christiaens GC: Fetal sex determination from maternal plasma in pregnancies at risk for congenital adrenal hyperplasia. Obstet Gynecol 2001; 98: 374-378.

6 Devaney SA, Palomaki GE, Scott JA, Bianchi DW: Noninvasive fetal sex determination using cell-free fetal DNA: a systematic review and meta-analysis. JAMA 2011; 306: $627-636$. 
7 Hill M, Lewis C, Jenkins L, Allen S, Elles RG, Chitty LS: Implementing noninvasive prenatal fetal sex determination using cell-free fetal DNA in the United Kingdom. Expert Opin Biol Ther 2012; 12(Suppl 1): S119-S126.

8 Lo YM, Hjelm NM, Fidler C et al: Prenatal diagnosis of fetal RhD status by molecular analysis of maternal plasma. N Engl J Med 1998; 339: 1734-1738.

9 van der Schoot CE, Hahn S, Chitty LS: Non-invasive prenatal diagnosis and determination of fetal Rh status. Semin Fetal Neonatal Med 2008; 13: 63-68.

10 Bombard AT, Akolekar R, Farkas DH et al: Fetal RHD genotype detection from circulating cell-free fetal DNA in maternal plasma in non-sensitized RhD negative women. Prenat Diagn 2011; 31: 802-808.

11 Daley R, Hill M, Chitty LS: Non-invasive prenatal diagnosis: progress and potential. Arch Dis Child Fetal Neonatal Ed 2014; 99: F426-F430.

12 Chiu RWK, Chan KCA, Gao Y et al: Noninvasive prenatal diagnosis of fetal chromosomal aneuploidy by massively parallel genomic sequencing of DNA in maternal plasma. Proc Natl Acad Sci USA 2008; 105: 20458-20463.

13 Bianchi DW, Platt LD, Goldberg JD, Abuhamad AZ, Sehnert AJ, Rava RP: Genome-wide fetal aneuploidy detection by maternal plasma DNA sequencing. Obstet Gynecol 2012; 119: 890-901.

14 Futch T, Spinosa J, Bhatt S, de Feo E, Rava RP, Sehnert AJ: Initial clinical laboratory experience in noninvasive prenatal testing for fetal aneuploidy from maternal plasma DNA samples: initial clinical laboratory experience with noninvasive prenatal DNA testing. Prenat Diagn 2013; 33: 569-574.

15 Porreco RP, Garite TJ, Maurel K et al: Noninvasive prenatal screening for fetal trisomies $21,18,13$ and the common sex chromosome aneuploidies from maternal blood using massively parallel genomic sequencing of DNA. Am J Obstet Gynecol 2014; 211: 365.e1-365.e12.

16 Allyse $M$, Minear $M$, Rote $M$ et al: Non-invasive prenatal testing: a review of international implementation and challenges. Int J Womens Health 2015; 7: 113-126.

17 Bustamante-Aragonés $\mathrm{A}$, Rodríguez de Alba $\mathrm{M}$, Perlado $\mathrm{S}$ et al: Non-invasive prenatal diagnosis of single-gene disorders from maternal blood. Gene 2012; 504: 144-149.

18 Lench N, Barrett A, Fielding S et al: The clinical implementation of non-invasive prenatal diagnosis for single-gene disorders: challenges and progress made: noninvasive prenatal diagnosis of single-gene disorders. Prenat Diagn 2013; 33: 555-562.

19 Bustamante-Aragones A, Gallego-Merlo J, Trujillo-Tiebas MJ et al: New strategy for the prenatal detection/exclusion of paternal cystic fibrosis mutations in maternal plasma. J Cyst Fibros 2008; 7: 505-510.

20 Debrand E, Lykoudi A, Bradshaw E, Allen SK: A non-invasive droplet digital PCR (ddPCR) assay to detect paternal CFTR Mutations in the cell-free fetal DNA (cffDNA) of three pregnancies at risk of cystic fibrosis via compound heterozygosity. PLoS One 2015; 10: e0142729.

21 Hill M, Twiss P, Verhoef Tl et al: Non-invasive prenatal diagnosis for cystic fibrosis: detection of paternal mutations, exploration of patient preferences and cost analysis. Prenat Diagn 2015; 35: 950-958.

22 Chitty LS, Mason S, Barrett AN et al: Non-invasive prenatal diagnosis of achondroplasia and thanatophoric dysplasia: next-generation sequencing allows for a safer, more accurate, and comprehensive approach: non-invasive prenatal diagnosis using nextgeneration sequencing. Prenat Diagn 2015; 35: 656-662.

23 Lam K-WG, Jiang P, Liao GJW et al: Noninvasive prenatal diagnosis of monogenic diseases by targeted massively parallel sequencing of maternal plasma: application to thalassemia. Clin Chem 2012; 58: 1467-1475.

24 New MI, Tong YK, Yuen T et al: Noninvasive prenatal diagnosis of congenital adrenal hyperplasia using cell-free fetal DNA in maternal plasma. J Clin Endocrinol Metab 2014; 99: E1022-E1030.

$25 \mathrm{Ma} \mathrm{D}, \mathrm{Ge} \mathrm{H}$, Li X et al: Haplotype-based approach for noninvasive prenatal diagnosis of congenital adrenal hyperplasia by maternal plasma DNA sequencing. Gene 2014; 544: 252-258.
26 Xu Y, Li X, Ge H et al: Haplotype-based approach for noninvasive prenatal tests of Duchenne muscular dystrophy using cell-free fetal DNA in maternal plasma. Genet Med 2015; 17: 889-896.

27 Lo YMD, Chan KCA, Sun H et al: Maternal plasma DNA sequencing reveals the genomewide genetic and mutational profile of the fetus. Sci Transl Med 2010; 2: 61ra91.

28 Parks M, Court S, Cleary S et al: Non-invasive prenatal diagnosis of Duchenne and Becker muscular dystrophies by relative haplotype dosage. Prenat Diagn 2016; 36: 312-320.

29 Lefebvre S, Bürglen L, Reboullet S et al: Identification and characterization of a spinal muscular atrophy-determining gene. Cell 1995; 80: 155-165.

30 Farooq Faraz Tariq, Holcik Martin, MacKenzie Alex: Spinal muscular atrophy: classification, diagnosis, background, molecular mechanism and development of therapeutics. Neurodegener Dis 2013; doi:10.5772/53800.

31 Prior TW, Nagan N, Sugarman EA, Batish SD, Braastad C: Technical standards and guidelines for spinal muscular atrophy testing. Genet Med 2011; 13: 686-694.

32 Ogino S, Wilson RB, Gold B: New insights on the evolution of the SMN1 and SMN2 region: simulation and meta-analysis for allele and haplotype frequency calculations. Eur J Hum Genet 2004; 12: 1015-1023.

33 O'Leary P, Maxwell S, Murch A, Hendrie D: Prenatal screening for Down syndrome in Australia: costs and benefits of current and novel screening strategies. Aust NZ J Obstet Gynaecol 2013; 53: 425-433.

34 Tabor A, Alfirevic Z: Update on procedure-related risks for prenatal diagnosis techniques. Fetal Diagn Ther 2010; 27: 1-7.

35 Norton ME, Brar H, Weiss J et al: Non-Invasive Chromosomal Evaluation (NICE) Study: results of a multicenter prospective cohort study for detection of fetal trisomy 21 and trisomy 18. Am J Obstet Gynecol 2012; 207: 137.e1-137.e8.

36 Jensen TJ, Zwiefelhofer T, Tim RC et al: High-throughput massively parallel sequencing for fetal aneuploidy detection from maternal plasma. PLoS One 2013; 8: e57381.

37 Dar P, Curnow KJ, Gross SJ et al: Clinical experience and follow-up with large scale single-nucleotide polymorphism-based noninvasive prenatal aneuploidy testing. Am J Obstet Gynecol 2014; 211: 527.e1-527.e17.

38 Scheffer H, Cobben JM, Matthijs G, Wirth B: Best practice guidelines for molecular analysis in spinal muscular atrophy. Eur J Hum Genet 2001; 9: 484-491.

39 Ehrich M, Deciu C, Zwiefelhofer T et al: Noninvasive detection of fetal trisomy 21 by sequencing of DNA in maternal blood: a study in a clinical setting. Am J Obstet Gynecol 2011; 204: 205.e1-205.e11.

40 Palomaki GE, Kloza EM, Lambert-Messerlian GM et al: DNA sequencing of maternal plasma to detect Down syndrome: an international clinical validation study. Genet Med 2011; 13: 913-920.

41 Canick JA, Palomaki GE, Kloza EM, Lambert-Messerlian GM, Haddow JE: The impact of maternal plasma DNA fetal fraction on next generation sequencing tests for common fetal aneuploidies: Fetal fraction and non-invasive testing. Prenat Diagn 2013; 33: 667-674.

(c) (i) This work is licensed under a Creative Commons Attribution 4.0 International License. The images or other third party material in this article are included in the article's Creative Commons license, unless indicated otherwise in the credit line; if the material is not included under the Creative Commons license, users will need to obtain permission from the license holder to reproduce the material. To view a copy of this license, visit http:// creativecommons.org/licenses/by/4.0/

(C) The Author(s) 2017

Supplementary Information accompanies this paper on European Journal of Human Genetics website (http://www.nature.com/ejhg) 